\title{
Relaciones Interpersonales y Sintomatología Ansiosa en Niños Víctimas de Maltrato Parental
}

\section{Interpersonal Relationships and Anxiety Symptoms in Children Victim of Physical Abuse}

\author{
Marisela Ramírez, Pamela Rocha y Ramón Castillo \\ Universidad Santo Tomás de Chile
}

(Recepción: Octubre 2002 - Aceptación: Marzo 2003)

\begin{abstract}
El artículo describe cómo la vivencia de maltrato al interior del hogar se asocia a niveles altos de sintomatología ansiosa y a relaciones interpersonales disfucionales al interior del colegio.

Se comparan las relaciones interpersonales en el colegio y los niveles de sintomatología ansiosa en un grupo de 30 niños maltratados por sus padres y un grupo pareado de 30 niños no maltratados.

Los maltratados presentan mayor ansiedad, retraimiento social y pasan desapercibidos en situaciones sociales como el estudio y el juego. Sin embargo, son escogidos para guardar un secreto. Se discute como esta dinámica al interior del colegio reforzaría su sintomatología ansiosa y el retraimiento social; que frecuentemente se asocian a las experiencias de maltrato en el hogar.

Palabras claves: Maltrato Infantil, Relaciones Interpersonales, Sintomatología Ansiosa.
\end{abstract}

\begin{abstract}
This paper describes how the experience of violence within the home is associated with high levels of anxiety symptoms and with dysfunctional interpersonal relationships in the school.

A group of 30 children, physically abused by their parents, is compared with a matched group of 30 non abused children in both interpersonal relationships in the school, and level of anxiety symptoms.

Abused children present major anxiety, social introversion and seem to pass unnoticed in social situations like study and game. Nevertheless, they are chosen to keep secrets.

The way the dynamic inside the school would reinforce anxiety symptoms and social introversion, frequently associated with the experience of physical abuse, is discussed.
\end{abstract}

Key words: Child Abuse, Interpersonal Relationships, Anxiety symptoms

\section{Introducción}

Diversos autores plantean que para definir maltrato es prioritario y necesario considerar al niño maltratado, en cuanto a los factores de vulnerabilidad que posea y la etapa evolutiva en que se encuentra. Sin embargo, concuerdan en que dicha conceptualización implicaría no sólo valorar al niño agredido sino también al adulto agresor con sus creencias, salud mental y estrés, a la trama familiar y al entorno sociocultural en que ocurre la interacción. En este sentido la conducta agresiva pasa a ser parte de una red de interacciones violentas que involucra más allá de la díada víctima-victimario (Richard, 1986; Novoa, De la Barra, Alvarez, Gilchrist, Alvarez \& Gamboa, 1992; Ministerio de Salud, 1994;

Correspondencia: Ramón Castillo G. Escuela de Psicología, Universidad Santo Tomás de Chile. Avda. Ejército Libertador 146 Santiago-Chile. (56-2) 3625000 . Email: rcastillo@ust.cl, http://www.ust.cl
Arruabarrena \& De Paúl, 1997; De Paúl \& Martínez, 2000).

En el presente trabajo se considerará puntualmente el maltrato físico, que alude a toda aquella acción no accidental por parte de un adulto, padres, que lleva consigo un alto potencial de provocar daño físico o enfermedad en el niño. Dentro de esta tipología de maltrato se encuentran dos categorías de violencia; las agresiones menos graves hacia el niño tales como: tirarle un objeto, empujarlo o zamarrearlo y pegarle una cachetada, y la violencia física grave que está definida por las conductas de golpear al niño con un objeto, darle una golpiza, quemarlo, amenazarlo con un arma o usarla para agredirlo (Haz, 1997).

Los criterios de esta definición están dados por una acción activa y física, que posee intencionalidad frente a otro que es vulnerable, y que implica consecuencias a corto plazo, tales como síndrome post-traumático; a mediano plazo, como la disminución del rendimiento; y largo 


\section{RAMÍREZ, ROCHA Y CASTILLO}

plazo, aspectos como el daño en la autoestima, sintomatología depresiva, repetición del patrón violento con los hijos y daño en la estructura de apego (Arruabarrena \& De Paúl, 1997; Horton \& Cruise, 1997).

Para comprender el maltrato infantil con todo lo que ello implica, se ha recurrido al modelo ecológico de Belsky (1980), ya que es un modelo integrativo que explica su etiología, no limitándose a la interacción hijo-padre, sino abarcando también al ambiente que rodea dicha interacción. Este identifica las variables de los diversos niveles, diferenciando entre factores que predisponen a la familia a desarrollar los malos tratos y factores protectores que lo limi$\tan$ e impiden.

El nivel ontogénico que corresponde a las características del individuo contribuyentes al maltrato, tales como la inteligencia, habilidades parentales, las características físicas y variables relativas a la propia crianza de los padres. El nivel de microsistema que incluye a los factores o circunstancias familiares que contribuyen a favorecer o impedir el maltrato; tales como la relación de los padres e hijos con sus propias características psicológicas y comportamentales. El nivel de exosistema que integra las influencias sociales de la comunidad en que se haya inserta la familia; tales como las relaciones sociales y el contexto ámbito laboral y escolar. Finalmente se encuentra el nivel de macrosistema, que agrupa a los determinantes socioculturales y macroeconómicos del maltrato (Belsky, 1980; Barudy, 1998, 2000; Cichetti, 1981; Corsi, 1994; De Paúl, 1994).

Desde una mirada evolutiva hay que decir que el niño se forma durante la primera infancia en función de las interacciones con su cuidador principal, en este caso con los padres o quien ejerza tal rol. Estas interacciones, van generando representaciones internas del niño, las cuales van a influir posteriormente en sus nuevas relaciones con el exosistema y en su capacidad para explorar y enfrentarse a situaciones posteriores nuevas y estresantes. De esta forma, las primeras experiencias entabladas durante la primera etapa de la vida son la base de la capacidad de entablar relaciones íntimas y emocionalmente saludables, que es lo que caracteriza al ser humano. Es de esta manera como el cariño, la empatía, el compartir, el inhibir la agresión y un sin número de otras características se asocian estrechamente con las capacidades nuclea- res del apego ocurridas durante la niñez temprana (Perry \& Runyan, 1998; Perry, 1999).

Los niños maltratados podrían desarrollar modelos internos de representación, que les haría difícil reconocer figuras de vínculo apoyadoras, incluso la conducta afectiva podría percibirse como hostil, lo que puede provocar reacciones defensivas o agresivas frente a determinadas situaciones, teniendo como resultado probablemente, una pérdida de oportunidad para desarrollar relaciones basadas en el apoyo y la confianza. De este modo, se infiere una disfunción o inadecuación en la interacción padrehijo, que se traduce en un fracaso de las pautas de socialización (Bowlby, 1973, 1980; en Gracia \& Musitu, 1993; Bowlby, 1986). De esta forma las experiencias negativas del niño con sus padres podrían estar explicando el desarrollo de problemas en el ajuste psicosocial (Gracia \& Musitu, 1993).

Se ha comprobado que la conducta de estos niños presenta diversos problemas, los cuales pueden manifestarse de manera interna o externa. Si el niño internaliza sus respuestas, el comportamiento tiende a caracterizarse por una pasividad, apatía, retraimiento social, bajo rendimiento escolar, sentimientos depresivos, conductas autodestructivas como forma de resolver problemas, así como también hábitos nerviosos o problemas somáticos. A la inversa, si el niño exterioriza su ansiedad y agresividad, su conducta puede estar caracterizada por la impulsividad, hiperactividad, desobediencia, conducta destructiva, falta de autocontrol y con frecuencia comportamientos violentos hacia otras personas y su entorno. En consecuencia se comportan de acuerdo a sus impulsos y no a las normas sociales establecidas (Almazan, Castro \& Vidal 1999; Rohner \& Rohner, 1980, en Gracia \& Musitu, 1993; Larraín, Vega \& Delgado, 1997; De Paúl \& Martínez, 2000).

De esta manera la literatura unido a la realización de diversos estudios postulan dos categorías para clasificar el comportamiento social de los niños que han sufrido maltrato: (a) La primera agrupa a los niños que tienen un comportamiento más bien pasivo, son niños obedientes y sumisos a las órdenes de sus padres, mostrándose tímidos, ariscos y esquivos en el contacto social. Así se postula que la causa de dicho comportamiento de sumisión a los deseos adultos se debería a que el niño tiene la convicción de que el amor es condicionado a las nece- 
sidades paternales y sólo con esta actitud el niño tendría protección y cuidado de sus padres dentro de un ambiente hostil. (b) La segunda categoría agrupa a aquellos niños con mayores tasas de agresión, frustración e impulsividad. Son niños que presentan un repertorio emocional limitado en cuanto a la expresión de sus sentimientos, les cuesta disfrutar y expresar placer en las actividades con otros niños, además presentan dificultad en la autorregulación de sus emociones y escasa empatía, y con frecuencia utilizan la agresión, presentando una conducta castigadora con sus pares, siendo hostiles y rebeldes, lo que a su vez los hace ser poco hábiles en la interacción y manejo de las relaciones interpersonales (Cerezo \& Frías, 1994; Inostroza \& Quitral, 1998; Kempe \& Kempe,1985; Richard, 1986; Gracia \& Musitu, 1993; Cantón y Cortes, 1997; Jiménez, 1997).

Además, el niño que ha sufrido maltrato físico siempre percibirá al adulto como el agresor y él como víctima, así se comprobó en un estudio realizado por Helfer y Kempe (1976) en donde observaron que los niños maltratados, entre 6 y 14 años de edad, no se relacionaban con los adultos con comodidad y confianza, presentándose ansiosos y temerosos frente a ellos; esta interacción social se caracteriza por la complementariedad (roles asimétricos con acciones propias de cada rol, por ejemplo: Mando/obedecimiento). Por tanto, éste adquiere una identificación con el papel de víctima frente a los adultos, lo cual es producto principalmente, de las constantes humillaciones a las que se ven sometidos por sus padres y del desarrollo de expectativas acerca de éstos como personas que no responden y no son disponibles, de los cuales el niño sólo espera el rechazo, lo que les hace tener un pobre concepto de sí mismos que es manifestado a través de conductas de aislamiento, apatía y agresividad, es así como, al relacionarse con sus pares ejerce el papel de agresor que ha sido aprendido de sus padres; esta segunda interacción social está caracterizada por la reciprocidad (roles y acciones intercambiables, ej: me agreden/agredo). Las manifestaciones conductuales agresivas de los niños maltratados, en la interacción con los de su edad, están determinados por diversas variables contextuales, es decir, no se comportan necesariamente de manera agresiva en todas las situaciones (Helfer \& Kempe, 1976; Straus \& Manciaux, 1982; Oates, 1986; Steele, 1986; Cantón \& Cortés, 1997).
Investigaciones realizadas, en donde compararon a niños maltratados y no maltratados físicamente, según las respuestas dadas por los padres en el Child Behavior Checklist (CBCL), los niños maltratados eran más agresivos y presentaban más problemas de conductas que los niños no maltratados. No obstante, la observación directa no reveló ninguna diferencia en la cantidad de comportamientos agresivos o no agresivos dirigidas a los miembros de la familia, ni en la gravedad de los problemas de conducta en niños maltratados y no maltratados (Cantón \& Cortés, 1997).

En algunos estudios efectuados sobre las relaciones entre iguales sugieren que los compañeros pueden mantener o incluso aumentar los problemas de los niños agresivos o retraídos, dando como resultado que los niños maltratados disminuyan sus intentos de interaccionar y consiguientemente se aíslen cada vez más y/o desarrollen formas más coercitivas de llamar la atención de sus iguales. Así mismo se comprobó por un estudio realizado por Salzinger, Feldman, Hammer, y Rosario (1993), que los niños maltratados durante la edad escolar, entre 8 y 12 años respectivamente, presentaban un bajo estatus entre sus iguales lo que se podría explicar por la percepción que tienen sus pares sobre la conducta social del niño (percibirlos como más agresivos y retraídos socialmente), así como por las distorsiones cognitivas que presentan los niños maltratados sobre las conductas, intenciones y sentimientos de sus iguales. De acuerdo con estos resultados estos autores propusieron un modelo teórico según el cual la violencia intrafamiliar suministra el contexto para el desarrollo de la conducta social desajustada y la comprensión inadecuada de las situaciones interpersonales dando como resultado un menor estatus social entre los compañeros y mal adaptación sobre un amplio rango de áreas sobre la conducta (Cantón \& Cortés, 1997).

Otro aspecto de interés respecto a las interacciones de los niños maltratados tiene que ver con la reacción de éstos ante las situaciones estresantes vivenciadas por sus pares, en donde se encontró que los pequeños maltratados frente a la angustia experimentada por otros niños de su edad (manifestada por llantos o miedo), reaccionaban de manera violenta además del desinterés prestado a su par y la poca empatía. Esta conducta opuesta a la prosocial, sería la responsable de algunos de los problemas inmediatos que sufren estos niños 


\section{RAMÍREZ, ROCHA Y CASTILLO}

abusados, tales como el rechazo de sus compañeros, y más largo plazo, como el maltrato a sus propios hijos (Gracia \& Musitu, 1993; De Paúl \& Martínez, 2000).

Se plantea como hipótesis que los niños maltratados físicamente por sus padres presentan, en comparación con los niños que no han recibido dicho maltrato, mayor rechazo por parte de sus compañeros o pares, mayor agresividad en la relación social, mayor sintomatología ansiosa, más retraimiento, inseguridad y pasividad las relaciones interpersonales.

\section{Método}

\section{Participantes}

La muestra fue seleccionada de un establecimiento educacional municipalizado de la comuna de Santiago, y estuvo constituida por $60 \mathrm{ni}$ ños, de los cuales 30 pertenecían al Grupo Niños Maltratados (NM) físicamente por sus padres, y los 30 restantes conformaban el Grupo Niños No Maltratados (NNM). La clasificación de ambos grupos se realizó a partir de los datos obtenidos por medio de Conflict Tactics Scale (CTS), la que permitió la identificación de maltrato físico parental. Efectuándose posteriormente, un pareamiento para la conformación del Grupo Niños No Maltratados, tomando en consideración las variables de segmentación tales como: edad, sexo, escolaridad y nivel socioeconómico. Esta muestra estuvo intencionalmente constituida por niños escolares entre los 9 y 15 años de edad ${ }^{1}$.

\section{Instrumentos}

Conflict Tactics Scale (CTS) de Straus (1990, en Haz \& Ramírez, 1996; 1998), adaptado para Chile por Larraín (1992), es un instrumento que evalúa las conductas utilizadas por los miembros de una familia para resolver los conflictos intrafamiliares.

1 Debido principalmente a los avances cognoscitivos y lingüísticos que estos poseen, obteniéndose así reproducciones más fidedignas de la realidad, ya que el niño se interesa por los aspectos reales dejando de lado las fantasías; la objetividad es el ideal en esta edad. Además, el lenguaje posee un mayor grado de conciencia y de reflexión (De Paúl \& Martínez, 2000). Con esto quedan saldadas aquellas variables que pudieran influir en nuestro estudio, como la fantasía que se haga el niño respecto de la relación con sus padres o del limitado vocabulario y capacidad lectora que el niño posea.
State-Trait Anxiety inventory for Children (STAIC); Inventario de Ansiedad Estado-Rasgo para niños de Spielberger, Gorsuch, y Lushene (1968), evalúa la sintomatología ansiosa en niños de 9 a 15 años.

Sociograma: Es una técnica que pretende poner de manifiesto los lazos de influencia y de preferencia que existen dentro de un grupo. Consiste en representar gráficamente las relaciones interpersonales en un grupo de individuos mediante un conjunto de puntos (individuos) conectados por una o varias líneas (relaciones interpersonales).

Child Behavior Check List (CBCL) de Achenbach y Edelbrock (1986). Es una escala que permite la observación y valoración de diversas conductas psicopatológicas y problemas de conductas componentes de la inadaptación escolar.

Los factores conductuales para hombre incluyen: conducta antisocial, agresividad-hiperactividad, ansiedad-depresión, quejas somáticas, crueldad-agresividad, depresión, ansiedad, inseguridad-pasividad, trastornos del desarrollo.

Los factores para las niñas incluyen: agresividad-hiperactividad, ansiedad-depresión, conducta antisocial, inmadurez social, confusión-retraimiento, conducta rebelde, quejas somáticas, inseguridad-pasividad, retraimiento.

Las variables de esta prueba, sobre la cual se baso el estudio, fueron en los niños la agresividad-hiperactividad, ansiedad-depresión, ansiedad y inseguridad-pasividad. En el caso de las niñas, se consideró la agresividad-hiperactividad, ansiedad-depresión, inseguridad-pasividad y retraimiento.

\section{Procedimiento}

Primeramente se revisaron los antecedentes de los niños a tratar en cuanto a la existencia o no de maltrato físico paterno, que se midió a través de la aplicación del CTS, conformando así el Grupo de 30 Niños Maltratados (NM), y por un Grupo de 30 Niños No Maltratados. El pareo se hizo en el mismo curso del niño, seleccionando al par que mostraba igualdad por edad, sexoy nivel socioeconómico. Luego se aplicó el STAIC que evalúa ansiedad estado y ansiedad rasgo, y Sociograma que permite ver la cantidad calidad, en cuanto a rechazo y aceptaciones, de las relaciones interpersonales establecidas por los niños. En una segunda fase, se aplicó el CBCL; el cual fue contestado por los padres de los niños, durante las reuniones de consejo. 


\section{RELACIONES INTERPERSONALES Y SINTOMATOLOGÍA}

\section{Resultados}

\section{Caracterización de la muestra}

El grupo se conformó con 60 niño pertenecientes a una escuela municipalizada del sector centro de la comuna de Santiago, de la Región Metropolitana. 38 de sexo masculino $(63,3 \%)$ y 22 de sexo femenino $(36,7 \%)$.

La escolarización de los niños se ubican en un rango de $4^{\circ}$ a $7^{\circ}$ básico. Las edades de estos fluctúan entre los 9 y 15 años, presentando un promedio de 11,7 y una desviación estándar de 1,07 . De estos 60 niños, 30 pasaron a conformar el Grupo Niños Maltratados (NM), los cuales presentan antecedentes de maltrato físico por parte de sus padres, y los 30 restantes pasaron a constituir el Grupo Niños No Maltratados (NNM), por medio de una pareo del cual resultó una total homogeneidad en las variables edad, sexo, escolaridad y nivel socio-económico entre los grupos a estudiar.

Dentro de los grupos 26 de los niños tenían 12 años (43,3\%). 16 niños, 11 años de edad $(26,7 \%) .8$ niños 10 años (13,3\%). 8 niños 13 años de edad $(13,3 \%)$. Dos niños del total tenía 15 años $(3,3 \%)$.
En cuanto al nivel de escolaridad, el $40 \%$ de los niños se encuentra en $6^{\circ}$ básico (24). Un $30 \%$ esta en $7^{\circ}$ básico (18). El 16,7\% se ubica en $5^{\circ}$ básico (10 niños); y el 13,3\% restante se encuentra en $4^{\circ}$ básico (8).

\section{Confiabilidad de los instrumentos}

La confiabilidad por consistencia interna de los instrumentos, estimada con el alfa de Cronbach fue de 0,97 para el inventario de $\mathrm{T}$. Achenbach. Para el inventario de ansiedad estado, la confiabilidad es 0,87 y la ansiedad rasgo es de 0,90. Para las subescalas de maltrato se obtuvieron valores que fluctuaron entre $0,45 \mathrm{y}$ 0,77 (ver tabla 1).

\section{Comparación de los grupos}

En cuanto a las variables medidas con el CTS (ver tabla 2); el grupo de niños maltratados reporta haber experimentado más maltrato físico y psicológico tanto del padre como de la madre, que el grupo de niños no maltratados.

Al comparar sólo los hombres en el cuestionario CBCL (ver tabla 3), no se observan diferencias los dos grupos, aun cuando con una sig-

Tabla 1: Confiabilidad de los instrumentos

\begin{tabular}{lc}
\hline Escalas & Alfa de Cronbach \\
\hline STAIC Ansiedad estado & 0,87 \\
STAIC Ansiedad rasgo & 0,90 \\
CBCL Total & 0,97 \\
CTS: Buen trato (padre) & 0,45 \\
CTS: Buen trato (madre) & 0,51 \\
CTS: Maltrato psicológico (padre) & 0,57 \\
CTS: Maltrato psicológico (madre) & 0,62 \\
CTS: Maltrato físico (padre) & 0,74 \\
CTS: Maltrato físico (madre) & 0,78 \\
\hline
\end{tabular}

Tabla 2: Comparación de medias en el CTS

\begin{tabular}{lccc}
\hline Variable & $\begin{array}{c}\text { Grupo } \\
\text { NNM } \\
\left(\mathrm{n}_{1}=30\right) \\
\text { Media (D.E.) }\end{array}$ & $\begin{array}{c}\text { Grupo } \\
\text { NM } \\
\left(\mathrm{n}_{2}=30\right) \\
\text { Media (D.E.) }\end{array}$ & \\
\hline Buen trato (Padre) & $12,07(3,54)$ & $11,89(4,14)$ & 0,177 NS \\
Buen trato (Madre) & $11,26(3,92)$ & $11,69(4,27)$ & $-0,392$ NS \\
Maltrato Psicológico (Padre) & $8,72(3,21)$ & $11,35(4,74)$ & $-2,375^{*}$ \\
Maltrato Psicológico (Madre) & $7,72(2,31)$ & $12,14(4,40)$ & $-4,719^{* *}$ \\
Maltrato Físico (Padre) & $6,32(0,67)$ & $10,38(4,27)$ & $-4,796^{* *}$ \\
Maltrato Físico (Madre) & $6,27(0,64)$ & $11,26(4,68)$ & $-5,499^{* *}$ \\
\hline
\end{tabular}

** $\mathrm{p}<0,01 ; * \mathrm{p}<0,05 ;$ NS no significativo 


\section{RAMÍREZ, ROCHA Y CASTILLO}

nificación de 0,054 los niños maltratados tienen levemente mayores puntuaciones en el factor de depresión que los no maltratados. Mientras que cuando se analiza sólo a las mujeres (ver tabla 4), la niñas maltratadas tiene puntuaciones más altas en el factor retraimiento que las no maltratadas; la misma tendencia se observa con el factor ansiedad depresión, aunque es con una significación 0,052 .

Respecto a las variables medidas por el STAIC (ver tabla 5), tanto en la ansiedad estado como en la ansiedad rasgo, el grupo de niños maltratados tiene puntuaciones más altas que el grupo de no maltratados.

En cuanto a las aceptaciones y rechazos, en el estudio, el juego y la posibilidad de contar un secreto, analizadas con el sociograma, el grupo de niños maltratados y el de no maltratados se asemeja bastante, aún cuando el de niños no maltratados tiene mayores rechazos ante la posibilidad de que alguien les cuente un secreto (ver tabla 6). En la tabla 7, es posible observar además que los alumnos de cursos inferiores $-4^{\circ} \mathrm{y}$ $5^{\circ}$ básico- tienden a aceptar en el juego más a los niños maltratados que los alumnos de $6^{\circ}$ y $7^{\circ}$ básico. Sin embargo, cuando se analizan los datos en su totalidad que hay una tendencia general a aceptar y rechazar a los niños no maltratados en el estudio y en el juego, en todos los cursos; áreas en las que los niños maltratados parecen invisibilizarse.

En síntesis, Los niños maltratados presentan mayores niveles de ansiedad estado y rasgo medidos con el STAIC. Concordante con lo anterior, en términos de las destrezas sociales medidas con el CBCL; las mujeres maltratadas tiene

Tabla 3: Comparación de medias en los factores del CBCL para hombres

\begin{tabular}{lcrc}
\hline Factores & $\begin{array}{c}\text { Grupo } \\
\text { NNM } \\
(\mathrm{n}=19)\end{array}$ & $\begin{array}{c}\text { Grupo } \\
\text { NM } \\
(\mathrm{n}=19)\end{array}$ & $\begin{array}{c}\text { Z de } \\
\text { Milcoxon }\end{array}$ \\
& $\begin{array}{c}\text { Media (D.E.) } \\
\text { Media (D.E.) }\end{array}$ & \\
\hline Conducta Antisocial & $6,57(4,39)$ & $7,71(8,21)$ & $-0,412 \mathrm{NS}$ \\
Agresividad - Hiperactividad & $16,77(7,61)$ & $16,83(10,56)$ & $-0,730 \mathrm{NS}$ \\
Ansiedad - Depresión & $7,88(4,20)$ & $10,15(4,80)$ & $-1,445 \mathrm{NS}$ \\
Quejas Somáticas & $3,12(2,76)$ & $2,86(3,07)$ & $-0,415 \mathrm{NS}$ \\
Crueldad - Agresividad & $2,33(1,75)$ & $2,18(3,49)$ & $-1,027 \mathrm{NS}$ \\
Depresión & $1,65(1,90)$ & $3,00(2,73)$ & $-1,925 \mathrm{NS}$ \\
Ansiedad & $3,73(1,93)$ & $3,28(1,75)$ & $-0,561 \mathrm{NS}$ \\
Inseguridad - Pasividad & $3,89(1,85)$ & $3,30(1,71)$ & $-0,957 \mathrm{NS}$ \\
Trastorno del Desarrollo & $1,78(1,34)$ & $1,96(1,84)$ & $-0,162 \mathrm{NS}$ \\
\hline
\end{tabular}

** $\mathrm{p}<0,01 ; * \mathrm{p}<0,05 ;$ NS no significativo

Tabla 4: Comparación de medias en los factores del CBCL para mujeres

\begin{tabular}{|c|c|c|c|}
\hline Factores & $\begin{array}{c}\text { Grupo } \\
\text { NNM } \\
(\mathrm{n}=11) \\
\text { Media (D.E.) }\end{array}$ & $\begin{array}{c}\text { Grupo } \\
\text { NM } \\
(\mathrm{n}=11) \\
\text { Media (D.E.) }\end{array}$ & $\begin{array}{c}\mathrm{Zde} \\
\text { Wilcoxon }\end{array}$ \\
\hline Agresividad - Hiperactividad & $15,81(7,25)$ & $16,61(10,01)$ & $-0,784 \mathrm{NS}$ \\
\hline Ansiedad - Depresión & $7,68(4,91)$ & $10,79(6,03)$ & $-1,940 \mathrm{NS}$ \\
\hline Conducta Antisocial & $9,89(5,09)$ & $9,16(8,06)$ & $-1,308 \mathrm{NS}$ \\
\hline Inmadurez Social & $6,68(2,98)$ & $8,04(5,20)$ & $-0,735 \mathrm{NS}$ \\
\hline Confusión - Retraimiento & $4,24(3,33)$ & $5,70(4,05)$ & $-1,141 \mathrm{NS}$ \\
\hline Conducta Rebelde & $4,43(2,38)$ & $5,43(4,21)$ & $-0,442 \mathrm{NS}$ \\
\hline Quejas Somáticas & $2,19(1,90)$ & $2,25(2,03)$ & $-0,571 \mathrm{NS}$ \\
\hline Inseguridad - pasividad & $4,85(2,19)$ & $3,96(2,49)$ & $-1,092 \mathrm{NS}$ \\
\hline Retraimiento & $1,23(0,90)$ & $1,73(0,78)$ & $-2,175 *$ \\
\hline
\end{tabular}


Tabla 5: Comparación de medias en el STAIC

\begin{tabular}{|c|c|c|c|}
\hline Variable & $\begin{array}{c}\text { Grupo } \\
\text { NNM } \\
\left(\mathrm{n}_{1}=30\right) \\
\text { Media (D.E.) }\end{array}$ & $\begin{array}{c}\text { Grupo } \\
\text { NM } \\
\left(\mathrm{n}_{2}=30\right) \\
\text { Media (D.E.) }\end{array}$ & $\mathrm{t}$ \\
\hline Ansiedad Estado & $10,54(5,74)$ & $15,65(8,94)$ & $-2,455 *$ \\
\hline Ansiedad Rasgo & $14,62(9,28)$ & $21,42(7,84)$ & $-2,857 * *$ \\
\hline
\end{tabular}

** $p<0,01 ; * p<0,05$; NS no significativo

Tabla 6: Comparación de medias en el sociograma

\begin{tabular}{lccc}
\hline Situación Social & $\begin{array}{c}\text { Grupo } \\
\text { NNM } \\
\left(\mathrm{n}_{1}=30\right) \\
\text { Media (D.E.) }\end{array}$ & $\begin{array}{c}\text { Grupo } \\
\text { NM } \\
\left(\mathrm{n}_{2}=30\right) \\
\text { Media (D.E.) }\end{array}$ & $\mathrm{t}$ \\
\hline Aceptación en el Juego & $1,61(0,70)$ & $1,47(0,70)$ & $0,599 \mathrm{NS}$ \\
Rechazo en el Juego & $3,27(3,08)$ & $1,69(1,18)$ & $1,830 \mathrm{NS}$ \\
Aceptación para el estudio & $2,24(1,92)$ & $1,69(1,14)$ & $0,988 \mathrm{NS}$ \\
Rechazo para el estudio & $3,00(3,30)$ & $1,62(0,96)$ & $1,459 \mathrm{NS}$ \\
Aceptación para un secreto & $1,31(0,48)$ & $1,36(0,66)$ & $-0,264 \mathrm{NS}$ \\
Rechazo para contar un secreto & $2,84(1,74)$ & $1,23(0,60)$ & $3,726 * *$ \\
\hline
\end{tabular}

$* * \mathrm{p}<0,01 ; * \mathrm{p}<0,05 ;$ NS no significativo

Tabla 7: Comparación de aceptaciones y rechazos en distintas situaciones sociales por curso

\begin{tabular}{|c|c|c|c|c|c|c|}
\hline $\begin{array}{l}\text { Curso y nivel } \\
\text { Situación Social }\end{array}$ & $7 \mathrm{~B}$ & $7 \mathrm{~A}$ & $6 \mathrm{~B}$ & $6 \mathrm{~A}$ & $5 B$ & $4 \mathrm{~A}$ \\
\hline Juego aceptado & $\mathrm{NNM}>\mathrm{NM}$ & $\mathrm{NNM}>\mathrm{NM}$ & $\mathrm{NNM}>\mathrm{NM}$ & $\mathrm{NNM}=\mathrm{NM}$ & $\mathrm{NNM}<\mathrm{NM}$ & $\mathrm{NNM}<\mathrm{NM}$ \\
\hline Juego Rechazado & $N N M>N M$ & $\mathrm{NNM}>\mathrm{NM}$ & $\mathrm{NNM}<\mathrm{NM}$ & $\mathrm{NNM}>\mathrm{NM}$ & $\mathrm{NNM}>\mathrm{NM}$ & $\mathrm{NNM}<\mathrm{NM}$ \\
\hline Estudio Aceptado & $\mathrm{NNM}=\mathrm{NM}$ & $\mathrm{NNM}<\mathrm{NM}$ & $\mathrm{NNM}>\mathrm{NM}$ & $\mathrm{NNM}<\mathrm{NM}$ & $\mathrm{NNM}>\mathrm{NM}$ & $\mathrm{NNM}>\mathrm{NM}$ \\
\hline Estudio Rechazado & $N N M=N M$ & $\mathrm{NNM}>\mathrm{NM}$ & $\mathrm{NNM}>\mathrm{NM}$ & $\mathrm{NNM}>\mathrm{NM}$ & $\mathrm{NNM}>\mathrm{NM}$ & $\mathrm{NNM}>\mathrm{NM}$ \\
\hline Secreto Aceptado & $\mathrm{NNM}<\mathrm{NM}$ & NNM $>$ NM & $\mathrm{NNM}>\mathrm{NM}$ & $\mathrm{NNM}<\mathrm{NM}$ & $\mathrm{NNM}<\mathrm{NM}$ & $\mathrm{NNM}=\mathrm{NM}$ \\
\hline Secreto Rechazado & $N N M>N M$ & $\mathrm{NNM}>\mathrm{NM}$ & $\mathrm{NNM}=\mathrm{NM}$ & $\mathrm{NNM}>\mathrm{NM}$ & $\mathrm{NNM}>\mathrm{NM}$ & $\mathrm{NNM}>\mathrm{NM}$ \\
\hline
\end{tabular}

Nota: Los NNM son más aceptados en el juego (3), más rechazados en el juego (4), más aceptados en el estudio (3), más rechazados en el estudio (5) y más rechazados en el secreto (5). Por otro lado, los NM son más aceptados en el secreto (3).

más indicadores de retraimiento social que sus pares no maltratadas; mientras que en el caso de los varones, no se observan diferencias significativas entre los niños maltratados y los no maltratados. Al analizar las puntuaciones de las interacciones social con el sociograma, se observa que los niños maltratados son menos rechazados para contar un secreto. Cuando se analizan la interacciones en términos gráficos, los niños no maltratados presentan una mayor cantidad de aceptaciones y rechazos en el juego y en el estudio; áreas sociales en las que los niños maltratados se hacen invisibles. Sólo en la situación del secreto, éstos aparecen como menos rechazados.

\section{Discusión}

Para el modelo ecosistémico, la familia es el contexto inmediato en donde se fomenta el desarrollo de ciertas habilidades sociales que se ponen de manifiesto en el escolar y en el adolescente a la hora de interactuar con el grupo de pares. Sin embargo, paradójicamente es el lugar donde ocurre la mayor parte de las conductas maltratadoras.

En este sentido, muchos investigadores concuerdan en que la relación establecida por niños maltratados por sus padres presentarían dos tipos de conductas: los comportamientos de tipo 


\section{RAMÍREZ, ROCHA Y CASTILLO}

agresivo, y por otro lado conductas de evitación ante la relación social (Cantón y Cortés, 1997; Jiménez, 1997). Lo anterior no alude necesariamente a que estos comportamientos sean mutuamente excluyentes.

Los resultados arrojados por este estudio, muestran estas diferencias sociales sobre todo en la variable retraimiento en mujeres; sin embargo no se observaron diferencias en las conductas agresivas entre los niños maltratados en comparación a las conductas de los niños no maltratados. Por ende, se avalaría la propuesta que señala que los niños maltratados físicamente por los progenitores serían más retraídos e inseguros frente al contacto social; en especial las niñas maltratadas.

Así mismo, hay estudios que señalan que los niños maltratados físicamente por sus padres tendrían un comportamiento más bien pasivo, obediente y sumiso a las órdenes paternas, lo que los haría ser tímidos, temerosos, ansiosos, y esquivos en el contacto social. Postulándose también que la causa de dicho comportamiento de sumisión a los deseos adultos se debería a que el niño tiene la convicción de que el amor es condicionado a las necesidades paternales y sólo con esta actitud el niño tendría protección y cuidado de sus padres dentro de un ambiente hostil (Gracia \& Musitu, 1993; Jiménez, 1976; Straus \& Manciaux, 1982; Oates, 1986; Steele, 1986; Cantón \& Cortés, 1997).

Es así como dependiendo del contexto en el cual el niño se desenvuelve y de las relaciones vinculares establecidas por los niños con sus padres, los niños asimilarían ésta relación y sin duda tenderían a repetir su actitud pasiva, sumisa y ansiosa frente a otro que ven como agresor.

Este tipo de conductas se verían reflejadas en el contacto con sus pares, en donde los niños maltratados adoptarían un comportamiento de sumisión y pasividad frente al resto, mediante el cual buscarían obtener aceptación de su grupo de iguales. Lo que estaría justificado por la presencia de ciertas deficiencias a nivel social manifestadas en el niño, el que tiende a relacionarse de una manera asimétrica con los demás (víctima-victimario). Estas deficiencias sociales se verían además reflejadas en una conducta retraída y en un comportamiento tímido; lo que mantiene un ciclo de apatía y retraimiento como respuesta al medio.

A partir de esta propuesta y en base a los datos arrojados por el estudio, se puede concluir que los niños maltratados físicamente por sus progenitores, tenderían a expresar sus problemas de una manera internalizada, es decir, sus conductas se caracterizarían por el retraimiento, apatía y ansiedad principalmente.

De este modo surge la inquietud respecto a como se expresan los sentimientos de rabia e impotencia que puedan tener hacia sus padres maltratadores, aún cuando no lo expresan comportamentalmente. No obstante, presentan sintomatología ansiosa ya sea como estado situacional, o a nivel más prolongado, como rasgo. Sobre todo si es la madre la que maltrata físicamente, lo que es respaldado por otros estudios que han concluido que los adolescentes objeto de abusos físicos tienden a presentar mayor cantidad de problemas emocionales, como ansiedad, depresión, trastornos somáticos y hostilidad, en comparación con otros sujetos no sometidos a abuso (Malinosky-Rummell \& Hansen, 1993; Mullen, Martin \& Anderson, 1996 en Cantón \& Cortés, 1997).

Suponiendo esta situación en niños maltratados, las relaciones amistosas se verían desfavorecidas y poco gratificantes para el niño, debido a su deficiente capacidad para interactuar, su poca capacidad empática y retraimiento. Esto a su vez podría estar relacionado con la ansiedad, justificada en su relación vincular con los padres con roles asimétricos, en donde el niño se ve así mismo como el que debe cumplir las ordenes y adopta una actitud pasiva y sumisa frente a los demás. Así, en los niños maltratados la probabilidad de desarrollar relaciones amistosas satisfactorias es mínima, debido a que la existencia de maltrato altera su desarrollo normal. Por otro lado, esta ansiedad se incrementaría, toda vez que no son considerados por sus pares, en las situaciones más importantes dentro del ámbito escolar, como lo son jugar y estudiar.

En resumen los niños maltratados presentan mayor ansiedad, retraimiento social y pasan desapercibidos ante sus pares en situaciones sociales como el estudio y el juego. Sin embargo, y debido tal vez a su reserva o retraimiento, son escogidos para guardar un secreto. Esta dinámica en las actividades más importantes del colegio reforzaría su sintomatología ansiosa y el retraimiento social que se encuentra asociada a las experiencias de maltrato experimentado al interior del hogar. 


\section{RELACIONES INTERPERSONALES Y SINTOMATOLOGÍA}

\section{Referencias}

Achenbach, T. M. \& Edelbrock, C. S. (1983). Manual for the Child Behavior Checklist and Revised Child Profile. Burlington, VT: University Associates in Psychiatry.

Almazan, S.; Castro, S. \& Vidal, C. (1999). Estudio comparativo entre niños maltratados físicamente y una muestra normativa de niños, de seis a doce años, con relación a la variable agresión. Tesis para optar al grado de licenciado en Psicología. Santiago de Chile, Universidad Diego Portales, Facultad de Ciencias Humanas. Escuela de Psicología.

Arruabarrena, M. I. \& De Paúl, J. (1997). Maltrato a los niños en la familia; evaluación y tratamiento. Madrid: Editorial Pirámide.

Barudy, J. (1998). El dolor invisible de la infancia; una lectura ecosistémica del maltrato infantil. Buenos Aires: Editorial Paidós.

Barudy, J. (2000). Maltrato infantil. Ecología social: Prevención y reparación. Chile: Editorial Galdoc.

Belsky, J. (1980). Child maltreatment an ecological integration. American Psycologist. 35, 320-335.

Bowlby, J. (1986). Vinculos afectivos, formación, desarrollo y pérdida. Madrid-España: Editorial Morata.

Cantón, J. \& Cortés, M. (1997). Malos tratos y abuso sexual infantil. Madrid-España: Editorial Siglo XXI.

Cerezo, M. A. \& Frías, D. (1994).Emotional and cognitive adjustement in abused children. Child abuse and neglect, 18 (11), 923-932.

Cichetti, D. (1981). Developmental perspective on the etiology, intergenerational transmission, and sequelae of child maltreatment. New directions for child development; 11, 31-35.

Corsi, J. (1994). Violencia familiar; una mirada interdisciplinaria sobre un grave problema social. Argentina: Editorial Paidos.

De Paúl, J. \& Martínez, A. (2000). Maltrato y abandono en la infancia. Barcelona-España: Martínez Roca Ediciones.

Gracia, E. \& Musitu, G. (1993). El maltrato infantil. Un análisis ecológico de los factores de riesgo. Madrid: Ministerio de Asuntos Sociales.

Haz, A. (1997). Menores víctimas de maltrato físico: un estudio sobre prevalencia y factores de riesgo en 1000 familias Chilenas. Psykhe, 6, (1), 47-56.

Helfer \& Kempe. (1976). Child Abuse and neglect, the family and the community. U.S.A.: Ballinger Publ.

Horton, C. \& Cruise, T. (1997). Clinical assessment of child victims and adult survivors of child maltreatment. Journal of counseling and development.76, 93-104.

Inostroza, C. \& Quitral, S. (1998). "Estudio descriptivo sobre los factores de riesgo asociados al maltrato infantil en un grupo de madres de bajo y alto riesgo en la población Manuel Plaza en la comuna de Buín". Memoria para optar al título de Psicólogo, Escuela de Psicología, Universidad Santo Tomás, Santiago, Chile.

Jiménez, M. (1997). Psicopatología infantil. II Edición, Alfije Ediciones.

Kempe, R. \& Kempe, H. (1985).Niños maltratados. Madrid: Editorial Morata.

Larraín, S.; Vega, J. \& Delgado, I. (1997). Relaciones familiares y maltrato infantil. UNICEF, Departamento de Psicología, facultad de Ciencias Sociales, Universidad de Chile, Chile: Editorial Calicanto.

Ministerio de Salud. (1994). Orientaciones técnicas y programáticas para la prevención y atención integral del maltrato infantil en los servicios de salud. Santiago, Chile.

Novoa, F.; De la Barra, F.; Alvarez, P.; Gilchrist, A.; Alvarez, N. \& Gamboa, R. (1992). Síndrome del niño maltratado. Revista chilena de Pediatría, 63 (2) 1-13. Sociedad Chilena de Psiquiatría. Santiago, Chile.

Oates, K. (1986). Child abuse and neglect what happens eventually. N.Y.-U.S.A: Brunner/Mazel. Publ.

Perry, B. \& Runyan, D. (1998). Formación de vínculos y el desarrollo del afecto en niños maltratados. Vol I, $\mathrm{N}^{\circ} 5$, disponible en: http://www. childtrauma.org

Perry, B. (1999). Formación de vínculos y el desarrollo de apego en niños maltratados, en línea. Disponible en: http:/ /www.childtrauma.org

Richard, S. (1986). El niño maltratado. Buenos Aires: Editorial Hvmanitas.

Salzinger, S., Feldman, R. S., Hammer, M., \& Rosario, M. (1993). The effects of physical abuse on children's social relationships. Journal of Child Development, 64(1), 169-187.

Spielberger C. D., Gorsuch R. L., \& Lushene R. E. (1968) STAI, Manual for the State-Trait Anxiety Inventory (Self Evaluation Questionnaire). Palo Alto, California. Consulting Psychologists Press, Inc. Adaptación española: Cuestionario de Ansiedad Estado-Rasgo. Madrid, TEA Ediciones; 1988.

Steele, B. (1986). Notes on the lasting effects of early child abuse throughout the life cycle. Child abuse and neglect, 10, 283-291.

Straus, P. \& Manciaux, M. (1982). L' Enfant maltraite. Paris-Francia: Editorial Fleurus. 